\title{
Clinical Aspects and Surgical Outcomes of Congenital Cholesteatoma in 93 Children: Increasing Trends of Congenital Cholesteatoma from 1997 through 2012
}

\author{
Hyun Soo Cho ${ }^{1}$, Hak Geon Kim¹, Da Jung Jung ${ }^{1}$, Jeong Hun Jang ${ }^{1}$, \\ Sang Heun Lee ${ }^{2}$, and Kyu-Yup Lee ${ }^{1}$ \\ ${ }^{1}$ Department of Otorhinolaryngology-Head and Neck Surgery, School of Medicine, Kyungpook National University, Daegu, \\ ${ }^{2}$ Department of Otorhinolaryngology-Head and Neck Surgery, Daegu Veterans Hospital, Daegu, Korea
}

Received April 19,2016

Revised June 27, 2016

Accepted July 18, 2016

\author{
Address for correspondence \\ Kyu-Yup Lee, MD \\ Department of Otorhinolaryngology- \\ Head and Neck Surgery, \\ School of Medicine, \\ Kyungpook National University, \\ 130 Dongdeok-ro, Jung-gu, \\ Daegu 41944, Korea \\ Tel $+82-53-420-5785$ \\ Fax +82-53-423-4524 \\ E-mail kylee@knu.ac.kr
}

Background and Objectives: The recent increase in the reported incidence of congenital cholesteatoma (CC) may be secondary to the widespread use of otoendoscopy as well as an increased awareness of these lesions among primary care physicians. However, little research about CC has been conducted in a large group of patients. This study aimed to analyze the clinical characteristics of CC including the annual number of patients, symptoms, age at diagnosis, stage and type of disease, surgical techniques, recurrence, and postoperative complications. Subjects and Methods: Retrospective chart review was performed for patients who met the inclusion criteria between January 1997 and June 2012. Results: Ninety-three patients underwent surgery for CC. The age at operation ranged from 12 months to 17 years (mean age, 6.1 years). The number of patients was less than 4 per year until 2005, but increased to more than 10 per year since 2008. CC was most commonly reported as an incidental finding (58.1\%). The operative procedures included the transcanal myringotomy approach (46.2\%), canal wall up mastoidectomy (37.6\%), tympanoplasty (8.6\%), and canal wall down mastoidectomy (7.5\%). The recurrence rate was $20.4 \%$ and the complication rate was $12.9 \%$. No patients with stage I CC had complications. Conclusions: This study showed that the incidence of CC has recently increased notably. Most patients with stage I and II CC were completely cured by transtympanic surgery, and complication and recurrence rates increased according to the extent of disease. Early detection of $\mathrm{CC}$ is important to facilitate minimally invasive surgery and to reduce complication and recurrence rates.

J Audiol Otol 2016;20(3):168-173

\section{Introduction}

Cholesteatoma of the middle ear is a keratin-filled anomaly that is found medial to the tympanic membrane. In 1986, Levenson, et al. [1] defined congenital cholesteatoma (CC) as a pearly white mass medial to a normal tympanic membrane in patients with a normal pars tensa and flaccida and no history of otorrhea, tympanic membrane perforation, or previous otologic procedures; cases with canal atresia, intra-membra-

This is an Open Access article distributed under the terms of the Creative Commons Attribution Non-Commercial License (http://creativecommons.org/licenses/by-nc/3.0/) which permits unrestricted non-commercial use, distribution, and reproduction in any medium, provided the original work is properly cited. nous, or giant cholesteatoma are excluded; and a prior history of otitis media is not a cause for exclusion. CC is not a rare disease, with an incidence of 0.2 to $1.5 \%$ [1] of all intracranial tumors and 3.7 to $24 \%$ of all cholesteatomas [2]. The treatment goals for $\mathrm{CC}$ are complete removal of the lesion, preservation and improvement of hearing, and prevention of recurrence. These goals can be achieved only by surgical intervention. The surgical techniques include tympanoplasty, canal wall up mastoidectomy, and canal wall down mastoidectomy, and the surgical approach is determined by factors such as the location, type, and stage of the lesion as well as by the surgeon's preference and experience. Unlike acquired cholesteatoma, $\mathrm{CC}$ does not disrupt Eustachian tube function 
and does not involve inflammation around the lesion. Therefore, surgery for $\mathrm{CC}$ should preserve function as much as possible by avoiding damage to the normal structures. We previously reported surgical outcomes in patients with early $\mathrm{CC}$ removed via transcanal myringotomy [3], and several other groups have published reports on CC, but large-scale studies are rare $[4,5]$.

The reported incidence of $\mathrm{CC}$ has tended to increase recently, most likely because of increased awareness of the lesion among primary care physicians, pediatricians, and otolaryngologists. Improvements in otoendoscopes and their widespread have also had an important role in early identification of CC [3]. However, the incidence of CC remains unclear. In this study, we reviewed 93 cases of $\mathrm{CC}$ that presented during 15 years at a single tertiary referral hospital and aimed to investigate the annual number of patients as well as the clinical features, surgical methods, and outcomes depending on the extent of disease.

\section{Subjects and Methods}

We performed a retrospective chart review of patients who were diagnosed with CC between January 1997 and December 2012 at the Department of Otorhinolaryngology, School of Medicine, Kyungpook National University, Daegu, South Korea. The study was approved by the Institutional Review Board of the Clinical Research Institute at Kyungpook National University Hospital, Republic of Korea (study 201208-003). The patients were diagnosed by using the criteria proposed by Levenson, et al. [1]. Classification of the stage of $\mathrm{CC}$ was determined according to the recommendations of Potsic, et al. [6] as follows: stage I, disease restricted to a single quadrant of the tympanic cavity; stage II, multiple quadrants affected, but no ossicular involvement or mastoid extension; stage III, disease with ossicular involvement; and stage IV, disease with mastoid extension. The type of CC was determined according to the classification of McGill, et al. [7] as closed cyst (encapsulated cholesteatoma) or open infiltrative cyst (cholesteatoma matrix in direct contact with the middle ear mucosa).

All patients underwent temporal bone computed tomography to confirm the presence of the tumor before surgery, and the final diagnosis of $\mathrm{CC}$ was confirmed by post-operative pathology examination. All patients were followed for at least 12 months after surgery. The patients who underwent surgery after July 2012 were excluded from this study because the follow-up period was not adequate. The patients' brief medical histories, physical findings, operative reports, comparisons of preoperative and postoperative radiologic findings, and fol- low-up records were reviewed. The presenting symptoms, distribution of tumor stage and type, year of operation, age at operation, surgery type, complications, and recurrence rates were analyzed.

\section{Results}

A total of 93 patients, 64 boys $(68.8 \%)$ and 29 girls (31.2\%), who were diagnosed with CC and underwent surgical treatment between January 1997 and June 2012 were included in the study. Right-sided CC and left-sided CC occurred in 52 and 41 patients, respectively. The patient characteristics are summarized in Table 1. The mean number of operations annually was 6.9 during the entire study period, and more than 10 operations were performed per year after 2008, showing a trend toward a rapid increase in comparison with the number of surgeries from 1997 to 2005 (Fig. 1). The mean age at operation was 6.1 years (range, 1 to 17 years). The age-specific occurrence rate was highest at 3 years (13.9\%) (Fig. 2). All of the patients who were diagnosed with $\mathrm{CC}$ by the age of 1 year had disease restricted to the middle ear without ossicular and mastoid involvement, while CC discovered in older children included tumors with ossicular extension (from the age of 2 years) or mastoid involvement (from the age of 3 years). Stage I CC occurred in 2 children at 12 years of age; however, most cases of stage I CC were found in children younger than 6 years of age. Stage III or IV CC was found only in children older than 7 years of age. Fifty-four patients $(58.1 \%)$ were diagnosed incidentally in primary care clinics and 35 patients $(37.6 \%)$ presented with conductive hearing loss (Table 1). Most stage I or II lesions were detected incidentally, while patients with advanced-stage (III or IV) tumors were referred for hearing impairment (Fig. 3). The tumor distribution according to stage is shown in Table 2; 32

Table 1. Demographic features

\begin{tabular}{lc}
\hline \multicolumn{1}{c}{ Characteristic } & Value \\
\hline Age at operation, mean (range) & $6.1(1-17)$ \\
Sex, numbers (\%) & \\
$\quad$ Male & $64(68.8)$ \\
Female & $29(31.2)$ \\
Ear, numbers (\%) & \\
$\quad$ Right & $52(55.9)$ \\
Left & $41(44.1)$ \\
Chief complaint, numbers (\%) & \\
Incidental finding & $54(58.1)$ \\
Hearing impairment & $35(37.6)$ \\
Otalgia & $3(3.2)$ \\
Facial palsy & $1(1.1)$ \\
\hline
\end{tabular}



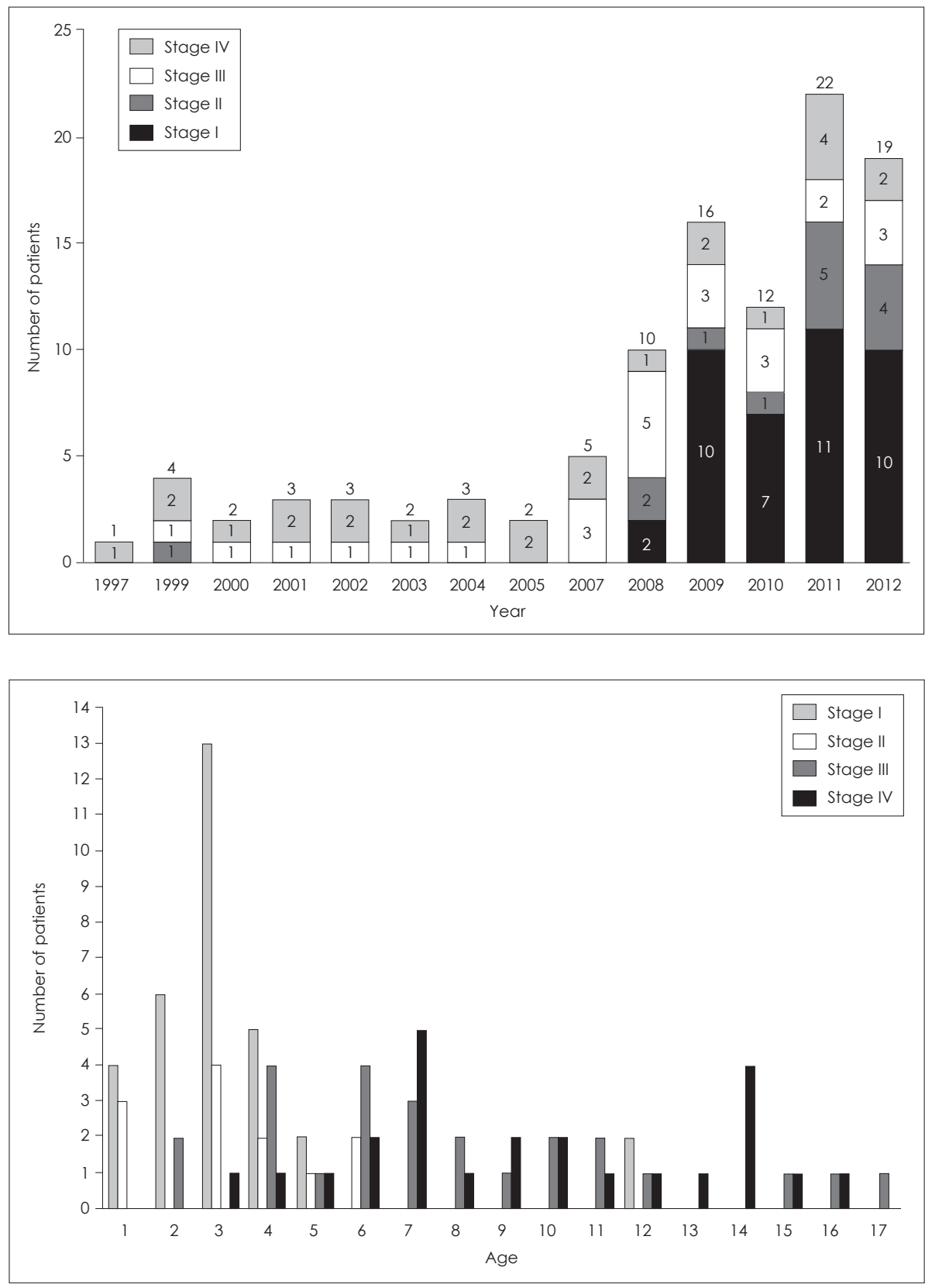

Fig. 1. Annual number of patients. The number on top of each bar indicates the total number of patients each year and the number in the bar indicates the number of patients in each category. patients $(34.4 \%)$ had stage I CC, 12 patients (12.9\%) had stage II CC, and 25 patients $(26.8 \%)$ had stage III or IV tumors. No type showed significant predominance. Closedtype $\mathrm{CC}$ and open-type $\mathrm{CC}$ were found in 47 and 46 patients, respectively. The tumors were most commonly found in the anterosuperior quadrant ( $n=35$ of $93,37.6 \%$ ). The transcanal myringotomy approach was the most frequently used surgical technique ( $n=43 ; 46.2 \%)$, followed by canal wall up mastoidectomy $(n=35 ; 37.6 \%)$, tympanoplasty $(n=8 ; 8.6 \%)$, and canal wall down mastoidectomy $(n=7 ; 7.5 \%)$ (Table 2$)$. Among patients with early-stage CC, 29 of 32 (90\%) with stage I disease and 10 of 12 (83\%) with stage II disease under- went surgery via transcanal myringotomy, while 19 of 25 patients (76\%) with stage III CC and 22 of 24 patients (91\%) with stage IV CC underwent classical mastoidectomy (Fig. 4). The mean follow-up duration after surgery was 45 months (range, 12 to 172 months). We defined recurrent cholesteatoma as a whitish mass found beyond the tympanic membrane or as pathologically confirmed cholesteatoma after revision surgery because of otologic symptoms such as otalgia and otorrhea during the follow-up period. Complications were defined as persistence of tympanic membrane perforation, hearing loss, otorrhea, or tinnitus 3 months after surgery. Nineteen patients (20.4\%) had recurrence (Table 3) and $12(12.9 \%)$ had 


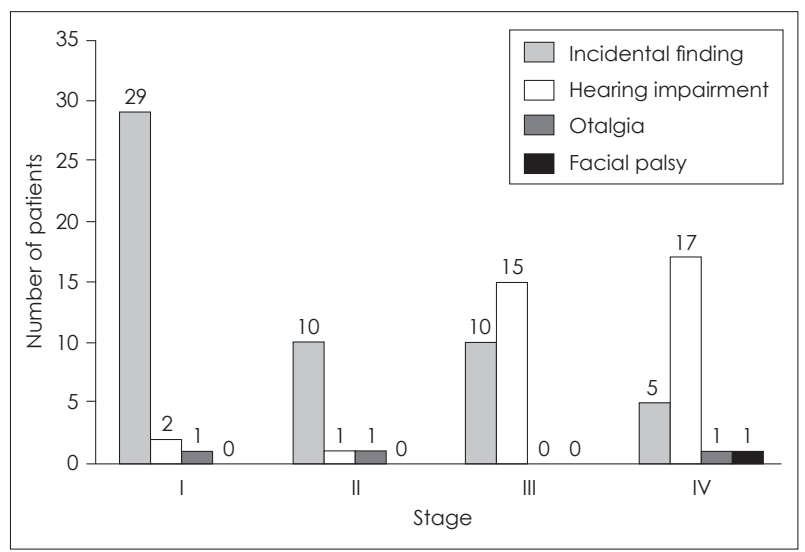

Fig. 3. Distribution of presenting symptoms according to disease stage. The number on top of each bar indicates the number of patients in each category.

Table 2. Disease type, location and operation method

\begin{tabular}{lc}
\hline \multicolumn{1}{c}{ Findings at surgery } & Numbers of patients (\%) \\
\hline Type of mass & $47(50.5)$ \\
Closed & $46(49.5)$ \\
Open & \\
Disease stage & $32(34.4)$ \\
I & $12(12.9)$ \\
II & $25(26.8)$ \\
III & $24(25.8)$ \\
IV & \\
Location & $35(37.6)$ \\
AS quadrant & $6(6.5)$ \\
PS quadrant & $1(1.1)$ \\
Al quadrant & $2(2.2)$ \\
PI quadrant & $10(10.8)$ \\
AS and PS quadrant & $14(15.1)$ \\
PS and PI quadrant & $9(9.7)$ \\
AS and Al quadrant & $16(17.2)$ \\
Multiple quadrant & \\
Operation method & $43(46.2)$ \\
Transcanal myringotomy approach & $8(8.6)$ \\
Tympanoplasty & $35(37.6)$ \\
Canal wall up mastoidectomy & $7(7.5)$ \\
Canal wall down mastoidectomy &
\end{tabular}

Multiple: more than three quarters. No.: number, AS: anterosuperior, PS: posterosuperior, Al: anteroinferior, PI: posteroinferior

complications, 8 of which (66\%) occurred in patients with stage IV open-type $\mathrm{CC}$ and included otorrhea $(\mathrm{n}=4)$, tinnitus $(n=2)$, otitis media with effusion $(n=1)$, and protrusion of an ossicular replacement prosthesis $(\mathrm{n}=1)$.

\section{Discussion}

This study showed that the number of patients with CC has

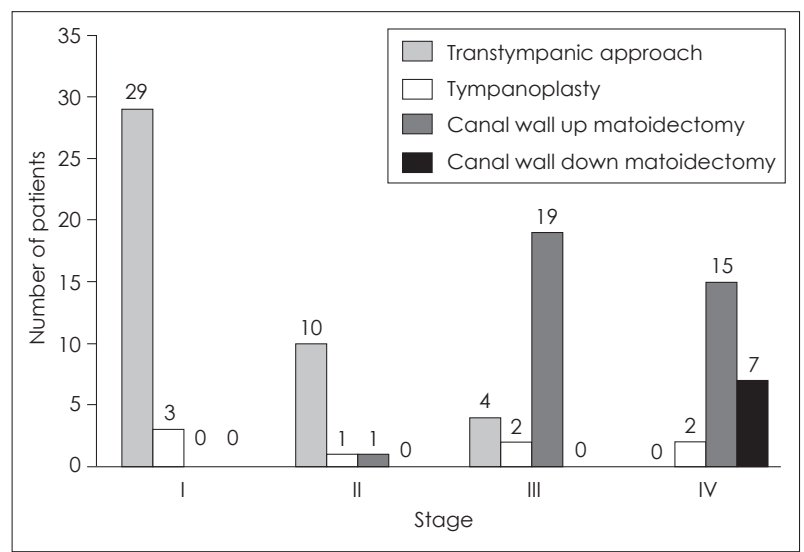

Fig. 4. Distribution of surgery types according to disease stage. The number on top of each bar indicates the number of patients in each category.

Table 3. The number of patients of recurrence and complications according to stage

\begin{tabular}{ccc}
\hline Disease stage & Recurrence $(\%)$ & Complication $(\%)$ \\
\hline I $(n=32)$ & $5(15.2)$ & $0(0)$ \\
II $(n=12)$ & $4(33.3)$ & $2(16.7)$ \\
III $(n=25)$ & $6(25.0)$ & $2(8.3)$ \\
IV $(n=24)$ & $4(16.7)$ & $8(33.3)$ \\
\hline Total & $19(20.4)$ & $12(12.9)$ \\
\hline
\end{tabular}

recently increased notably, and this might be due to early diagnosis and increased awareness of the lesion. The data shown in Fig. 1 depict the rapid increase in CC since 2008, with an especially a high percentage of early-stage CC. Before 2008, the number of patients with this disease was small and the proportion of advanced-stage $\mathrm{CC}$ was high. We hypothesize that it is difficult to diagnose whether $\mathrm{CC}$ is congenital or acquired in cases of advanced-stage $\mathrm{CC}$ because perforation of the tympanic membrane may occur in advanced-stage $\mathrm{CC}$, which could result in advanced-stage $\mathrm{CC}$ being misdiagnosed as acquired type of cholesteatoma. Therefore, the number of patients with CC may be lower than the actual state of the past.

Given the increasing awareness and concern for early diagnosis of CC, House and Sheehy [8] recommended that CC be considered a possibility in patients with unilateral otitis media with effusion or inexplicable unilateral conductive hearing loss. Further, Sana and Zini [9] emphasized early detection of the disease by using otoscopic, audiologic, and radiologic evaluations for children with conductive hearing loss, and suggested a screening program for school children. In the present study, the number of patients with $\mathrm{CC}$ was greatest at the age of 3 years $(n=18)$, and the age distribution suggests that the rate of $\mathrm{CC}$ detection increases with age up to 3 years and gradually decreases thereafter. Younger age at de- 
tection was also associated with an earlier stage of disease. Patients with stage I CC accounted for the highest proportion in each age group until the age of 5, while all of the patients with CC discovered after the age of 7 years had stage III or IV disease, with the exception of 2 patients with stage I disease at 12 years of age. No patients had stage IV CC at the age of 1 or 2 (Fig. 2). The distribution of surgical approaches implies that less-invasive surgery is possible with an early diagnosis (or an early stage).

The possibility of detecting asymptomatic $\mathrm{CC}$ in pediatric patients has recently increased in South Korea because of the extended awareness of CC among general practitioners and advanced medical technologies such as the otoendoscope. As a result, our findings show that the number of patients with $\mathrm{CC}$ in Korea has rapidly increased since 2007.

In terms of presenting symptoms, $\mathrm{CC}$ was asymptomatic and was found incidentally in most cases (58.1\%), and conductive hearing loss was the presenting symptom in $37.6 \%$ of the patients. Among the patients with conductive hearing loss, 32 (91.4\%) had stage III or IV CC (Table 1). On the other hand, 30 of the 33 patients in whom CC was found incidentally had stage I lesions. In 29 of these cases, the tumors were removed completely by using the transcanal myringotomy approach (Fig. 4), which is less invasive than previous methods, requires short operative times and hospital stays, and can usually be performed by otolaryngologists who are experienced in myringotomy [3]. The transcanal myringotomy approach is particularly beneficial for pediatric patients because it is easy to manage with no special treatment after surgery. This approach does not involve visible scarring or complications, and sequelae occur rarely. In the present study, only 12 of the 93 patients had complications, 8 of whom had stage IV CC. The remaining 4 complications were in patients with stage II and III tumors and included persistent tympanic membrane perforation $(n=2)$, intermittent otorrhea $(n=1)$, and hearing impairment $(n=1)$ (Table 3$)$. Only 1 patient who underwent transcanal myringotomy surgery had complications. The patient had stage II CC and had persistent tympanic membrane perforation after surgery. Otherwise, the tympanic incision spontaneously healed within 3 to 11 weeks (average, 5 weeks) in 42 of 43 patients in the transtympanic surgery group.

Pediatric $\mathrm{CC}$ tends to be more aggressive and extensive than $\mathrm{CC}$ in adults, and it is associated with a higher rate of recurrence $[10,11]$. According to the report of Potsic, et al. [12] the recurrence rate after surgery was $13 \%$ among patients with stage I CC and 25\% among those with stage II CC, while Stapleton, et al. [13] reported that $5 \%$ of patients with stage I CC and 24\% with stage II CC had recurrence. In our study,

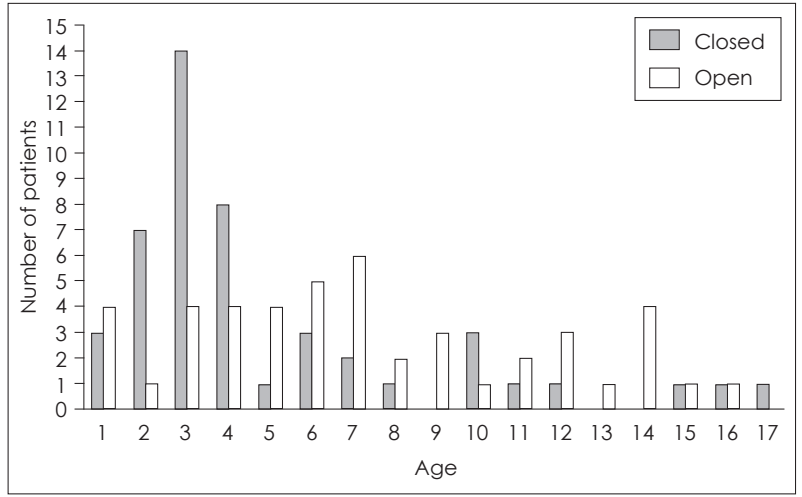

Fig. 5. Distribution of tumor types (open or closed) according to age.

the recurrence rate was $15.2 \%$ in patients with stage I CC, $33.3 \%$ in those with stage II CC, $25 \%$ among patients with stage III CC, and $16.7 \%$ in patients with stage IV CC (Table 3 ). The higher recurrence rate of stage III compared with stage IV is because 4 cases of stage III CC were treated only by the transcanal myringotomy approach, and half of these cases recurred. On the other hand, no cases with stage IV who underwent canal wall down mastoidectomy experienced recurrence. This suggests that extensive surgery is needed to lower the recurrence rate in advanced-stage cases. In stage II $\mathrm{CC}, 4$ cases recurred, and 3 of them were removed by using the transcanal myringotomy approach.

As for the type of CC, closed-type occurred more commonly among patients aged 2 to 4 years, while open-type occurred more commonly after the age of 5 (Fig. 5). The age distribution of closed-type lesions was similar to the distribution of stage I lesions, and open-type CC was evenly distributed at all ages.

In terms of location, the most common site of $\mathrm{CC}$ with a single quadrant was the anterosuperior quadrant of the tympanic membrane ( $n=35$ of $93,37.6 \%$ ), which is consistent with a previous study [14]. Regarding quadrant involvement, 44 patients $(47.3 \%)$ had single-quadrant involvement and 49 patients $(52.7 \%)$ had two- or multiple-quadrant involvement (Table 2).

In conclusion, this study included 93 patients who underwent surgical treatment for CC. Our findings show that the number of operations performed for $\mathrm{CC}$ in Korea has rapidly increased. The most common site of $\mathrm{CC}$ was the anterosuperior quadrant of the tympanic membrane. The majority of patients who had stage I CC diagnosed incidentally required only transtympanic surgery and recovered completely, which is an indication of the importance of early diagnosis. Further, transtympanic surgery has a low complication rate. Our results suggest that the transcanal myringotomy approach is a good option for treatment of stage I CC. Further research 
should be directed toward early diagnosis because surgery that is more extensive is required with advanced stages and pediatric $\mathrm{CC}$ tends to be aggressive.

\section{Conflicts of interest}

The authors have no financial conflicts of interest.

\section{REFERENCES}

1) Levenson MJ, Michaels L, Parisier SC. Congenital cholesteatomas of the middle ear in children: origin and management. Otolaryngol Clin North Am 1989;22:941-54.

2) Friedberg J. Congenital cholesteatoma. Laryngoscope 1994;104(3 Pt 2):1-24.

3) Lee SH, Jang JH, Lee D, Lee HR, Lee KY. Surgical outcomes of early congenital cholesteatoma: minimally invasive transcanal approach. Laryngoscope 2014;124:755-9.

4) Kim DK, Kim HM, Suh MW, Lee JH, Oh SH, Kim CS, et al. Analysis of risk factors for the occurrence of residual cholesteatoma after congenital cholesteatoma surgery. Korean J OtorhinolaryngolHead Neck Surg 2008;51:120-4.

5) Yoo SW, Kwon SY, Kim HJ. Risk factors of post-operative recidivism in congenital cholesteatoma of the middle ear. Korean J Otorhinolaryngol-Head Neck Surg 2014;57:664-70.
6) Potsic WP, Samadi DS, Marsh RR, Wetmore RF. A staging system for congenital cholesteatoma. Arch Otolaryngol Head Neck Surg 2002;128:1009-12.

7) McGill TJ, Merchant S, Healy GB, Friedman EM. Congenital cholesteatoma of the middle ear in children: a clinical and histopathological report. Laryngoscope 1991;101(6 Pt 1):606-13.

8) House JW, Sheehy JL. Cholesteatoma with intact tympanic membrane: a report of 41 cases. Laryngoscope 1980;90:70-6.

9) Sanna M, Zini C. "Congenital cholesteatoma" of the middle ear. A report of 11 cases. Am J Otol 1984;5:368-73.

10) Fageeh NA, Schloss MD, Elahi MM, Tewfik TL, Manoukian JJ. Surgical treatment of cholesteatoma in children. J Otolaryngol 1999; 28:309-12.

11) Stern SJ, Fazekas-May M. Cholesteatoma in the pediatric population: prognostic indicators for surgical decision making. Laryngoscope 1992;102(12 Pt 1):1349-52.

12) Potsic WP, Korman SB, Samadi DS, Wetmore RF. Congenital cholesteatoma: 20 years' experience at The Children's Hospital of Philadelphia. Otolaryngol Head Neck Surg 2002;126:409-14.

13) Stapleton AL, Egloff AM, Yellon RF. Congenital cholesteatoma: predictors for residual disease and hearing outcomes. Arch Otolaryngol Head Neck Surg 2012;138:280-5.

14) Kazahaya K, Potsic WP. Congenital cholesteatoma. Curr Opin Otolaryngol Head Neck Surg 2004;12:398-403. 